\title{
THE AETIOLOGY AND MANAGEMENT OF CHRONIC IDIOPATHIC FACIAL PAIN: A REVIEW
}

\author{
ZA Abdul Rahman. The Aetiology and Management of \\ Chronic Idiopathic Facial Pain: A Review, Annals Dent \\ Univ Malaya 1998; 5: 40-44
}

\begin{abstract}
Chronic idiopathic facial pain is the diagnosis given to a group of orofacial pain of psychogenic origin which includes atypical facial pain, facial-arthromyalgia, atypical odontalgia and oral dysaesthesia. Despite various biochemical findings, the condition remains poorly understood, but we have begun to understand the nature of these patients. This review discusses the possible aetiology of the disease through various biochemical and clinical findings. The contribution of behavioural and psychological factors to the clinical course of the disease are described. The type of adverse life events that predispose people to the disease and their potency are briefly mentioned. The current diagnostic approach for the disease is also mentioned. Treatment includes antidepressant medication, physiotherapy, bite-guards and analgesics. The problems encountered in the long-term management and outcome studies of these patients include drop-outs, non-compliance and denial.
\end{abstract}

Keywords: Chronic facial pain, facial arthromyalgia, aetiology, management

\section{Introduction}

Patients with emotional problems may present with the following physical symptoms in the oro-facial region (1):

i) Pain over the temporomandibular joints and associated muscles, known as facial-arthromyalgia.

ii) Pain in the non muscular part of the face termed atypical facial pain.

iii) Pain in the teeth or atypical odontalgia.

iv) Oral dysaesthesia which includes burning tongue and taste disturbances.

v) Tension headache

vi) Migraine

vii) Facial migrainous neuralgia.

These groups of symptoms are currently called as Chronic idiopathic facial pain (International Association for Study of Pain).

Facial pain offers a challenge to the present day clinician in terms of diagnosis and choice of treatment. It has been of interest to many medical and dental specialities including oral and maxillofacial surgery, oral medicine, restorative dentistry, otorhinolaryngology, psychiatry, and neurology. Genuine collaboration between the specialities hardly exists. The results of the facial pain audit study conducted in 1990 advocated the setting up of an interdisciplinary facial pain clinic consisting of surgeons or physicians and the liaison psychiatrists (2). Previous work on the long term outcome of facial pain. treatment has suggested that future research should address the questions as to who should treat the patients, with what and for how long (3).

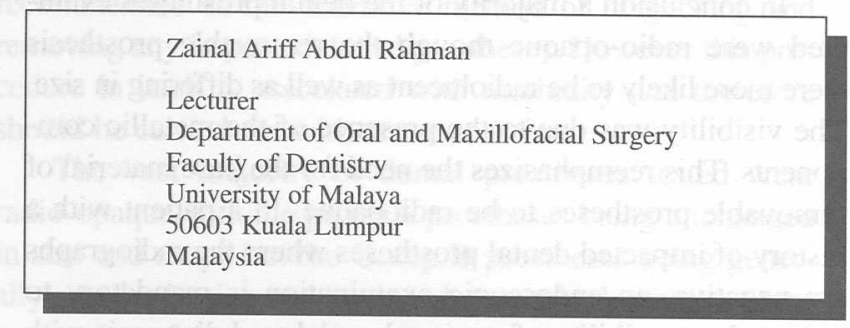

\section{Aetiology}

Patients with an increased tendency to react to stress, adverse life events and psychopathological disorders are vulnerable to chronic idiopathic facial pain (1). Stressful life events have been strongly associated with chronic facial pain (4). However recent studies have illustrated that there was no significant difference in desirable and undesirable life events in Temporomandibular joint pain patients as compared to the control group (5). However the concept of vulnerability of this group of patients has been suggested and that these patients have poor tolerance to stress (6).

The vulnerability concept was further supported by the existence of common symptoms at different ages of patients that suffered from facial pain. During childhood these patients may have had abdominal or ear pain. At adolescence they may experience TMJ pain or dysmenorrhea. Later on they may have irritable bowel, neck pain, back pain and pruritus $(7,18)$.

Recent studies have increased our understanding on the biochemical basis of the disease. The beta endorphins with other endogenous opioid are now believed to play major a role in mediating pain-modulation network. The analgesic effect of beta endorphins have been shown to be antagonised by adrenocorticotropic hormone(ACTH) which is released in high amounts during stress (9). The level of cortisol in the blood of chronic idiopathic pain patient has been reported to be significantly high (10).

A deficiency of conjugated tyramine sulphate in urine which commonly occurs in patients with endogenous depression have also been found in facial pain patients who were not depressed (11). Other biochemical markers such as serotonine metabolites in cerebrospinal fluid(12), abnormal dexamethasone suppression test and low serum and urine melatonin levels have also been confirmed (13). It has also been discovered that there is increased levels of Leukotriene B4 and 15-METE in saline aspirates of painful temporomandibular joint (14).

Depression is another factor associated with chronic idiopathic facial pain. It has been recently estimated that 30 $60 \%$ of these patients suffer from depression (15). In a study on patients attending the facial pain clinic(16) about $41.3 \%$ have been found to have depressive disorders. Other psychiatric problems encountered in this study are somatoform or psychogenic pain disorders (30.4\%), non- affective psychosis $(7.3 \%)$ and anxiety disorders and alcoholism (12.3\%) (16). It has also been suggested that depression can be a cause and also a consequence of facial pain (17). 


\section{Stress factors}

Stress experienced by facial pain patients can be in the form of negative or adverse life event such as bereavement, a congenitally deformed child or family illness. It can also be in the form of positive life events like promotion, moving into a new house and marriage. Other chronic adversities found in facial pain patients include unhappy marriage, work or social isolation, children with parental strife and difficulties at school (18).

In a study on patients with psychogenic facial pain, organic pain, psychogenic bodily symptoms and organic disease it was found that patients with psychogenic pain had a greater prevalence of the following early experiences:

1) Parents who are physically or verbally abusive of each other.

2) Parents who are abusive of the child

3) Children who deflected aggression from one parent to the other onto themselves.

4) Parents who suffered from an illness or other painful conditions.

5) Parent of the same gender with the patient suffering from pain.

6) Patient had the same location of pain as the parent.

7) Numerous surgeries during adulthood

8) Disturbed social and working life (19).

Major life changes and multiple daily hassles have been identified as initiators for stress responses (17). Individuals who were susceptible to the chronic pain problem belonged to the group which experienced major life changes as a threat to the physical or psychologic integrity. Recent researches have clarified that daily life hassles can also cause physical illness which includes chronic facial pain (17).

Patients with a psychiatric problem termed as post traumatic stress disorder have been shown to have increased susceptibility for chronic facial pain(20). The stressors that cause this syndrome have been shown to be very distressing and patients usually experienced them with intense fear, terror and helplessness. A study carried out on a group of 34 chronic idiopathic facial pain patients showed that $15 \%$ of these patients have post traumatic stress disorders (20).

\section{Management of facial pain}

\section{Diagnosis}

The diagnosis of Chronic Idiopathic Facial Pain is achieved by proper history and thorough examination. Pertes et al (21) suggested that the following information must be gathered from the history:

1) The chief complaints.

2) Location of the pain.

3) Characteristic of the pain i.e quality, intensity, mode of onset and temporal behaviour.

4) A chronological account of the complaint which includes the results of previous examinations and also the current treatment.

5) Associated symptoms.

6) The factors that aggravate, precipitate or relieve the pain.
7) Personal and past medical history.

Difficulty in accomplishing these is due to lack of courage, time and experience by the general or dental practitioner to take a detailed medical, family and social history. They may also fail to identify the psychogenic aspects of the problem. These difficulties have to be overcome to avoid unnecessary dental, surgical and medical investigations and treatment (18).

Detailed enquiry into the chief complaint is essential as this will provide a good working diagnosis since orofacial pain has specific characteristics in terms of onset, its chronicity, intensity, quality, location and associated signs and symptoms (22). Chronic idiopathic facial pain is defined as pain that persists for more than 3 month (25). It occurs after a traumatic and stressful episode and usually affecting the individuals within the age of 20 to 40 years (23). Facial arthromyalgia associated with nocturnal bruxism may also be present when the patient wakes up (24).

Chronic idiopathic facial pain also tends to be dull in character, radiates widely and are often bilateral (18). Atypical odontalgia commonly presents as a dull, aching, throbbing or burning pain (26). Inability of the patient to localise the pain can be indicative of a pychogenic pain (25).

Myofascial pain especially of the head and neck is usually accompanied by a series of other signs and symptoms in the form of pain, coincidental pathological conditions and psychosocial problems (27). Fricton (27) further documented a list of additional symptoms discovered in myofascial pain of the head and neck. These are classified into four groups:

1) Neurologic symptoms like tingling sensation, numbness, blurred vision, twitches, trembling and excess lacrimation

2) Gastrointestinal symptoms such as nausea, constipation, indigestion, diarrhoea and vomiting.

3) Musculoskeletal symptoms in the form of fatigue, tension, still joints and swelling.

4) Otologic symptoms which include tinnitus, ear pain, dizziness, vertigo fullness in the ear and diminished hearing.

Other symptoms include scratchy sensation, skin flushing, muscle twitching, hyperaesthesia, teeth sensitivity, increased salivation, excessive sweating and skin flushing (28-30).

Harris et al (18) suggested that enquiry into the presence of other symptoms like headache, neckache, backache, abdominal pain, pruritus and irritable bowel is essential in taking a facial pain history. Other stress-related disorders such as peptic ulceration, asthma and hypertension have also been shown to be present in post traumatic stress disorder patients (31).

When taking a medical history it has been suggested that a detailed enquiry of every system is essential (25). The complete family and social history is important in diagnosing facial pain. Patients should be asked about recent changes in the family life and at work. Events such as bereavement, serious illness of next of kin, physical abuse and divorce should be elicited (22). 
Clinical examination of facial pain patients includes inspection? palpation and a neurological examination. The movement, appearance and vital signs should be observed (22).The presence of neurological signs like ataxia, limping and leg weakness should raise the clinician's suspicion of an intracranial lesion (32). Patients with migraine have been said to be well-dressed and meticulously groomed with a list of their pain symptoms and medications taken $(33,34)$. Patients suffering from psychogenic headaches may appear calm and relaxed while describing the presence of severe disabling pain (17 and 25). Trigeminal neuralgia patients may turn up with unwashed face or unshaven (35).

The examination of vital signs like temperature and blood pressure may rule out fever and hypertension which can also cause facial pain. An increase in pulse rate may indicate that the patient is anxious or nervous (22).

The structures of the head and neck should be palpated for tenderness. The tenderness within facial muscles may suggest myalgia. Clicking, popping and crepitus on temporomandibular joint indicate internal derangement or degenerative changes of the temporomandibular joint (36). Examination of the cranial nerve for sensory disorders is essential in the patients with atypical facial pain.(18)

Intra oral examination includes palpation and percussion of the teeth and their supporting structures to rule out any dental pathology. Ridging of the buccal mucosa and lateral border of the tongue should be detected to establish bruxism. The inter incisal opening should also be recorded and any jaw deviation on opening and closing should be noted (18)

Investigations should include plain radiographs, biochemical tests, and an orthopantomogram. Computerised tomography scan is essential when there is paraesthesia or facial weaknesses (18). Patients with burning mouth syndrome should be tested for deficiences in serum vitamins B1, B2, B6 and B12, iron and glucose tolerance(37).

\section{Treatment}

Antidepressants have been successful in treating chronic idiopathic facial pain. However the action is not dependent on the antidepressant action of the drug (38). The knowledge on the mechanism of action of anti depressants in relieving facial pain has not been fully established.

There is a suggestion that chronic idiopathic facial pain and depressive illnesses have a common biological predisposition (39). This has further supported by the discovery of a common marker in these group of patients. The marker is a deficiency in the urinary excretion of conjugated tyramine. The sharing of a common marker may provide an explanation as to how antidepressants also function in controlling facial pain (11)

A 4-year follow up of facial pain patients treated with prothiaden (Dothiepine) with counselling showed that $75 \%$ improved(3). The tricylics have been thought to raise brain serotonin level which is involved in central analgesia (18)

Tricyclic antidepressants that are commonly used nowadays is nortryptyline. The non- depressed patient must be informed about the other effects of the antidepressants such as its analgesic and muscle relaxant properties. If the tricylics are ineffective the monoamine oxidase inhibitors like tranylcypromine are alternatives. One of the advantages of anti depressants in treating chronic pain is that they are non addictive. More recently selective serotonin reuptake inhibitors such as fluoexetine has been widely used on facial pain patients who were intolerable to the side effects of tricyclic antidepressant (18).

The other more common mode of treatment of facial arthromyalgia includes reassurance, occlusal splint, cognitive therapy, tranquillisers, jaw exercise, shortwave diathermy, ultrasound and surgery. Atypical facial pain and atypical odontalgia are often treated with reassurance, anti-inflammatory drugs, tranquillisers and cognitive therapy.

The use of iron, vitamin B12 and folic acid supplements to control oral dysaesthesia had been previously reported $(40,41)$. However most of the recent studies have indicated a low prevalence of nutritional deficiencies in patients with this condition. However there is only one study which showed a significant number of burning mouth syndrome patients with vitamins B1, B2 and B6 deficiencies in compared to the control group (42).

The protocol observed in the management of facial arthromyalgia patients is that the patients are reassured on the first visit if the pain is mild to moderate. $\sim$ printed hand-out explaining to the patients of their condition may reinforce the reassurance.

Should the problem persists an occlusal stabilization appliance may be prescribed. The role of the appliance in controlling the pain remains unexplained(18). Following failure of the use of the appliance, the patient may be prescribed antidepressants. The failure of the antidepressants to provide any improvement should lead to the use of arthroscopy. Arthrotomy may be carried out in cases where arthroscopy has failed to control the problem. The next step is to refer the patient to the liaison psychiatrist if the pain still persists. Consultation with a liaison psychiatrist should also take place earlier if the patient has been shown to have overt or latent emotional problems. At this stage if the pain still persists the patients may be subjected to group or cognitive therapy (18).

Psychological'treatment like behavioural therapy, relaxation, biofeedback therapy and hypnosis, and cognitive therapy can be helpful (38). Recently efforts have been made to incorporate cognitive and affective within behavioural therapy itself. Cognitive-behavioral therapy has often been used in multidisciplinary pain management(17). In this line of treatment both the patient and the therapist agree on how the pain problem is maintained by cognitive-behavioural factors. Both will also agree, on how cognitive-behavioural treatments can either remove the dysfunctional aspects of the problems or improve coping skills (17).

\section{Problems in treatment}

The problems that are encountered in the treatment and outcome studies of chronic pain include relapse, failure to enter treatment, drop outs from treatment and non- compliance. Studies on the follow up of chronic facial pain have shown the relapse to range from $30 \%$ to $60 \%$. Many researchers 
have attempted to identify the patient's characteristics. which contribute to relapse. However the result has been less than enlightening (42). Intractable pain among these patients may also be as a result of speculative surgical treatment of the condition. The basis of this mechanism can be explained by a phenomenon now termed as "windup"(43).

Lack of preparation of the patient to adjust to the natural environment and entry into social and occupational life may increase the risk of relapse (44). There is also the possibility of some inherent patient's characteristics like personality patterns, litigation or compensation status and psychopathology that contribute to relapse (42).

The factors shown to contribute to non-compliance were individual differences, nature of the disease, characteristic of the prescribed regimes, health-care provider-patient relationship and patient circumstances (42).

The chronic pain patients have a very high rate of failure to enter treatment. There are various reasons for these. This group of patients are often viewed as poorly motivated, resistant, denying and having poor prognosis (45). Interviews conducted on patients who fail to enter treatment had gathered the following reasons for it:

a) Lack of insurance coverage

b) Opposition of the spouse

c) Unwillingness to be hospitalised

d) Transportation difficulties

In addition there is also a general lack of interest in the focus of the programme (46).

The drop-out rate is a sign)ficant problem, regardless of which hospital or which country the patient is being treated. The range of drops out rate collected from various studies on chronic pain patients is between $5 \%$ to $39 \%$. These are reported by Beekman and Axtel (47) in a study to determine the causes of dropping-out. In this study the reasons of dropout that had been uncovered were:

a) Family problems (29\%)

b) Disruptive or uncooperative behaviour (23\%)

c) Financial difficulties (10\%)

d) Taking a job (6\%)

e) Other reasons $\{13 \%)$

f) Unspecified reasons (19\%).

It has been suggested that the best predictors for the dropping-out of temporomandibular disorders were social problems especially the attitude of others toward the patients and their complaints. The patient who gained less support from others were more likely to remain in treatment (48). Among other reasons for dropout that had been being suggested were the patients disatisfaction with treatment and lack of motivation to rehabilitate (49).

\section{Conclusion}

Chronic idiopathic facial pain poses major challenges to our daily clinical practices and it requires a special consideration in their management. Despite the fact that the exact aetiology remains unknown the understanding on the biochemical nature of the disease has been improved. Most of the patients were predisposed to adverse life events. There is a need for multidisciplinary set up for the management of this problem.
The diagnosis of the disease requires a proper detailed history and examination. This will prevent unnecessary invasive investigations. However other serious conditions like a space-occupying lesion within the cranium needs to be ruled out prior to diagnosis of chronic idiopathic facial pain. The various modes of treatment found to be effective include counselling, antidepressant, bite-guard, arthroscopy and cognitive behavioural therapy. The problems associated in the management of this group of patients include drop-out, non-compliance, relapse and failure to enter treatment.

\section{Reference}

1. Feinmann C. Psychiatric aspect of the mouth and face. Recent Advances in Clinical Psychiatry 1992; (7): 139147.

2. Ahmed K. Development of facial pain audit. M Sc thesis, University of London 1990.

3. Feinmann $\mathrm{C}$. The long term outcome of facial pain treatment. J Psychosomatic Res 1993; 37: 381-387.

4. Speculand B. Hughes A O. Gross A N. Role of stressful life experiences in the onset of TMJ pain dysfunction. Comm Dent Oral Epidemiol 1984; 12: 197202.

5. Marbach J J, Lennon M C, Dohrenwend B P. Candidate risk factors for temporomandibular pain and dysfunction syndrome: Psychosocial, health behaviour, physical illness and injury. Pain 1988; 34: 139-151.

6. Southwell J. Deary I I, Geissler P. Personality and anxiety in temporomandibular joint syndrome patients. J Oral Rehab 1990; 17: 239-243.

7. Feinmann C, Harris M, Cawley R. Psychogenic facial pain: presentation and management. Br Med J 1984; 228:436-438.

8 Engel G L. "Psychogenic" pain and pain prone patient. Am J Med 1959; 196: 129-136.

9. Smoke T. Fields H L. ACTH (1-24) blocks opiate induce analgesia in the rat. Brain Res 1980; 212: 202-206.

10. Blumer D, Zorick F. Heilbronn M, Roth T.Biological markers for depression in chronic pain. J Nerve Ment Dis 1982; 170: 425-428.

11. Aghabeigi B. Wasil M, Hannah P. Goodwin B. Feinmann C, Glover B. Sandler M, Harris M. Tyramine conjugation deficit inpatient with chronic idiopathic temporomandibular joint and orofacial pain. Pain 1993; 54:159-163.

12. Almay B G L, Hagendal J. von Knorring A, Oreland L. 5- HIAA and HVA in CSF in patients with idiopathic pain disorders. Biol Psychiatry 1987; 22: 403412.

13. von Knorring L. Pathogenesis of chronic pain syndromes. Nord. Psykiat. Tidsskr 1989; 43(suppl.20): 35 43.

14. Quinn J H. Bazan N G. Identification of protaglandins E2 and Leukotriene B4 in the synovial fluid the painful, dysfunctional temporo mandibular joint. J Oral Maxillofac Surg 1990; 38:968-971.

15. Magni G. On the relationship between chronic pain and depression when there is no organic lesion. Pain 1987; 31: 1-21.

16. Hughes A M, Hunter S, Still D, Lamey P J . Psychiatric 
disorders in a dental clinic. Br Dent J 1989; 166: 16-19.

17. Grzesiak R C. Psychologic considerations in temporomandibular dysfunction. Dent Clin N Am 1991; 35: 209-226

18. Harris M, Feinmann C, Wise M et al. Temporomandibular joint and orofacial pain: Clinical and medicolegal management problems. Br Dent J 1993; 174: $129-136$.

19. Adler R H, Zlot S, Hurny C et al. Engel's " Psychogenic pain and pain-prone patient": A retrospective, controlled clinical study. Psychosom Med. 1989; 52:87-101.

20. Aghabeigi B. Feinmann C, Harris M. Prevalence of post-traumatic disorder in patients with chronic idiopathic facial pain. Br J Oral Maxillofac Surg 1992;31: 360-364.

21. Pertes R A, Heir G M. Chronic orofacial pain: A practical approach to differential diagnosis. Dent Clin N Am 1991; 35: 123-140.

22. Balciunas B A, Siegel M A, Grace E G. A clinical approach to the diagnosis of facial pain. Dent Clin N Am 1992; 36; 987-1000.

23. Moss R A, Garrett J C. Temporomandibular joint dysfunction syndrome and myofascial pain dysfunction syndrome: A critical review. J Oral Rehabil 1984; 11:3.

24. Attanasio R. Nocturnal bruxism and its clinical management. Dent Clin N Am 1991; 32:245.

25. Rome H P, Harness D M, Kaplan H J. Psychologic and behavioural aspects of chronic facial pain. In Jacobson A L, Donlon W C (eds): Headache and facial pain. New York Raven Press 1990: 25.

26. Graf- Radford S B, Goldberg W K. Atypical odontalgia. Calif Dent Assoc J 1986; 14:27.

27. Fricton J R. Clinical care for myofascial pain. Dent Clin N Am 1991; 35 (1): 1-28.

28. Fricton J R, Kroening R, Haley D. Myofascial pain Syndrome: A review of 168 cases. Oral Surg 1982; 60: 615-623

29. Travel J. Identification of myofascial trigger point syndromes: Case of atypical facial neuralgia. Arch Psys Med Rehab 1981; 62: 100-106.

30. Travell J, Simons D G. Myofascial pain and dysfunction: The trigger point manual. Baltimore, Williams and Wilkins 1983: 63-158.

31. Lamey P J, Allam B F. Vitamin status of patients with burning mouth syndrome and the respond to replacement therapy. Br Dent J 1986; 81: 15

32. Lavyne M H, Patterson R M. Headache associated with brain tumor. In Dalesio D J (ed): Wolf's Headache and
Other Head Pain. flew York, Oxford University Press, 1987: 112.

33. Diamond S. Freitag F G. Solomon G D, et al. Migraine headache. Postgrad Med 1987; 81:174.

34. Saper J L. Migraine, migraine variants and related vascular headaches. In Jacobson A L, Donlon W C (eds): Headaches and Facial Pain. New YORK Raven Press, 1990: 81.

35. Donlon W C, Jacobson A L, Truta M P. Neuralgias. In Jacobson A L, Donlon W C (eds): Headache and Facial Pain. New York, Raven Press, 1990: 127.

36. Mahan P E, Alling CC. Temporomandibular joint arthritides. Facial Pain, Philadelphia, Lea and Febiger 1991: 219.

37. Grushka M, Sessle B J. Burning Mouth syndrome. Dent Clin N Am 1991; 35: 171-184.

38. Hunter S. The management of "psychogenic" orofacial pain. Br Med J 1992; 304: 329- 330.

39. Feinmann C. Pain relief by antidepressants: Possible modes of action. Pain 1985; 23: 1-8.

40. Afonsky D. Deficiency glossitis. Oral Surg 1951; 4:482.

41. Brooke R I, Segansky D P. Aetiology and investigation of the sore mouth. Can Dent Assoc J 1977; 10:504

42. Turk D C, Rudy T E. Neglected topics in the treatment of chronic pain patients - relapse noncompliance and adherence enhancement. Pain 1991; 44: 5-28.

43. Harris M. The surgical management of idiopathic facial pain produces intractable iatrogenic pain. Br J Oral Maxillofacial Surg 1996; 34: 1-3.

44. Blackwell B. Galbraith J R. Dahl D S. Chronic pain management. Hosp Commun Psychiat 1984; 35: 999-1008.

45. Turk D C, Rudy T E. Neglected factors in chronic pain treatment outcome studies-referral patterns, failure to enter treatment, and attrition. Pain 1990; 43: 7-25.

46. Sturgis E T. Schaefer A A, Sikora T L. Pain center follow-up study of treated and untreated patients. Arch Phys Med Rehab 1984; 65: 301-303.

47. Beekman C E, Axtell L. Ambulation, activity level, and pain. Outcomes of a program for spinal pain. Phys Ther 1985; 11: 1649-1657.

48. Funch D P. Gale E. Predicting treatment completion in behavioural therapy program for temporomandibular pain. J Psychosom Res 1986; 30: 57-62.

49. Gottlieb H. Strite L, Koller R. Madorsky A, Hockersmith V, Kleeman M, Wagner J. Comprehensive rehabilitation of patients having chronic low back pain. Arch Phys Med Rehab 1977; 58:101-108. 